\title{
The Weighed Core Symptom Scale and Prediction of ADHD in Adults - Objective Measures of Remission and Response to Treatment with Meth- ylphenidate
}

\author{
Hanna Edebol ${ }^{1, *}$, Lars Helldin ${ }^{2}$ and Torsten Norlander ${ }^{3,4}$ \\ ${ }^{1}$ Nutrition Gut Brain Interactions Research Centre, Örebro University, Örebro, Sweden \\ ${ }^{2}$ Department of Psychiatry, the NU-Health Care, Trollhättan, Sweden \\ ${ }^{3}$ Division of Psychology, Department of Clinical Neuroscience, Karolinska Institutet, Solna, Sweden \\ ${ }^{4}$ Evidens Research and Development Center, Göteborg, Sweden
}

\begin{abstract}
Objective: Two measures of the response rate and the optimal treatment response for adult ADHD were evaluated using methylphenidate. The hypotheses were that Prediction of ADHD (PADHD) defines remission, the Weighed Core Symptom (WCS) scale registers direct effects of medication and that WCS may indicate the optimal dose level during titration.

Design: PADHD and WCS were analyzed at baseline and after intake of low doses of either short-acting or modifiedrelease formulations of methylphenidate, MPH (Study I), during titration with modified-release formulations of MPH $(18 / 27,36,54,72 \mathrm{mg})$ and at three months follow-up (Study II).

Patients: Study I consisted of 63 participants (32 females) and Study II consisted of 10 participants (6 females) diagnosed with ADHD and who was to start with treatment.

Outcome measures: Prediction of ADHD (PADHD) indicates the occurrence of ADHD (No, Yes) and the Weighed Core Symptom scale (WCS) quantifies ADHD from 0 to 100 (max-min).

Results: The number of clinical cases of ADHD decreased after methylphenidate treatment according to PADHD. WCS increased $(p<0.001)$ from $9.75(S D=12.27)$ to $47.50(S D=29.75)$ with about $10 \mathrm{mg}$ of methylphenidate $(N=63)$. During titration, symptoms improved after $18 / 27 \mathrm{mg}$ and $36 \mathrm{mg}$ of methylphenidate and baseline-follow up comparisons showed WCS increments $(p=0.005)$ from $31.00(N=10, S D=26.85)$ to $69.00(N=10, S D=22.34)$.

Conclusions: PADHD defined remission and WCS measured therapeutic effects of methylphenidate in adult ADHD.
\end{abstract}

Keywords: Objective measures, Weighed Core Symptom scale, Prediction of ADHD, remission, ADHD.

\section{BACKGROUND}

Better long-term follow-up of treatment effectiveness with regard to predefined goals for Attention Deficit Hyperactivity Disorder (ADHD) among adults is warranted. Remission [1] is a criterion for treatment effectiveness and has been applied onto a number of psychiatric disorders in order to provide a rationale for the study and application of objective, measurable and clinically interpretable therapeutic endpoints. The goal for treatment of ADHD in adults ought to be full remission but the exact definition and measurement of such improvements has just recently begun to evolve.

ADHD affects around $5 \%$ of the adult population [2], including core symptoms of hyperactivity, inattention and impulsivity that constitute one out of three possible diagnostic

*Address correspondence to this author at the Nutrition Gut Brain Interactions Research Centre, Örebro University, Örebro, Sweden;

Tel: +46 (0) 732707 624; Email: hanna.edebol@oru.se subtypes [3]. Enormous societal costs, individual suffering and risks are associated with the disorder [4]. ADHD is also associated with severe under-treatment despite a massive body of cumulated research validating evidence-based treatments [5]. In order to gain as good a picture of ADHD symptom improvement during treatment as possible, one may employ both behavior ratings like the DSM-IV criteria [3] and more objective laboratory testing. One problem with rating scales is that they are built upon subjective observation and therefore likely to include biases but they are minimized when including also objective evaluation.

Monitoring treatment-efficacy involves evaluating the response to treatment defined as improvements in symptoms as a result of treatment [1]. The clinical trial response for ADHD has usually been set at $15-30 \%$ improvement in symptom scores $[6,7]$. In many cases, a patient who responds to this extent no longer meets the diagnostic criteria but since the initial level of symptoms is not accounted for there will also be responders who still have significant amounts of symptom and illness. In this regard, remission 
Table 1. Behavior Ratings.

Means $(M)$ and Standard Deviations $(S D)$ for Behavior Ratings Performed by Male and Female Participants with ADHD in Study I

\begin{tabular}{|c|c|c|c|c|c|}
\hline & & Males $(n=31)$ & & Females $(n=32)$ & \\
\hline & $\operatorname{Max}$ & $M$ & $S D$ & $M$ & $S D$ \\
\hline \multicolumn{6}{|l|}{ Behavior ratings } \\
\hline WURS 25 & 100 & 50.29 & 20.43 & 51.66 & 16.14 \\
\hline WRASS & 140 & 77.52 & 28.11 & 78.53 & 38.87 \\
\hline ASRS-Total score & 72 & 40.00 & 17.04 & 46.34 & 11.12 \\
\hline ASRS-Screener & 24 & 14.00 & 6.39 & 16.66 & 4.40 \\
\hline CAARSS & 78 & 30.06 & 24.50 & 38.59 & 26.10 \\
\hline
\end{tabular}

means "sufficient improvement" and has recently been introduced for ADHD [8] even though there is yet no universal agreement upon the criteria for remission in ADHD. In addition, females seek treatment to a higher extent than males, which suggests that there may be sex differences in the severity and course of the disorder. Recent meta-analytic work of laboratory measures in children with ADHD reported gender differences [9] and the response rate and optimal treatment response may be affected by gender differences as well.

Studies of goal-directed management with first-line treatment for ADHD demonstrate satisfactory results and patients may respond to the point that they become essentially asymptomatic and clinical criteria are no longer fulfilled [10-12]. Steele and colleagues [13] suggest that remission in ADHD be defined as "a loss of diagnostic status, minimal or no symptoms, and optimal functioning when individuals are being treated with or without medication" and further that "symptomatic remission can be operationalized as a mean total score of $\leq 1$ on most standardized questionnaires". There have been similar proposals of working definitions for remission in especially childhood ADHD based on cut-offs from behavior ratings like the Swanson Nolan And Pelham- version IV [14], the ADHD rating scale- version IV [15] and the Clinical Global Impression scale [16]. However, the use of quantifiable metrics with objective outcomes and standardized interpretation permit reliable estimation of treatment effectiveness and comparisons that may be of great value for the goal of remission.

The aim of the present study was to evaluate two prospective and objective measures of response and remission in adult ADHD using first-line treatment agencies for the disorder, i.e., methylphenidate. The instruments have been evaluated in terms of predictive power and the ability to quantify total amount of ADHD core symptoms in adults $[17,18]$. The negative predictive value range was $95-96 \%$, the positive predictive value range was 57-63\%, sensitivity $86-87 \%$ and specificity $83-85 \%[17,18]$.

Measures are derived from the Quantified Behavior Test Plus, Qb-Test-Plus, a laboratory test specialized into the measurement of ADHD core symptoms in adulthood [1921]. Previous studies suggest that the most reliable measure are attained from combining core symptom measures of hyperactivity, inattention and impulsivity into the composite Weighed Core Symptoms scale, WCS. The scale has been standardized with regard to adults with well-defined ADHD, non-ADHD normative participants, ADHD normative participants [17] and with regard to other psychiatric disorders presenting core symptoms of ADHD, e.g., bipolar II disorder, borderline personality disorder and participants with a disconfirmed ADHD-diagnosis [18]. The other instrument of interest for the present study is also derived from the QbTest-Plus and generates a categorical variable "Prediction of ADHD" (PADHD) that identifies clinical ADHD with good predictive power in a majority of cases $[17,18]$.

The aim was to evaluate WCS and PADHD in terms of response to treatment and remission during treatment with methylphenidate (MPH). We hypothesize that PADHD will indicate remission and that WCS will be sensitive to dose level changes, i.e., response, as well as to the clinically defined optimal dose level, i.e., remission.

In study I we evaluate the ability of WCS and PADHD to indicate response to treatment with short-acting MPH treatment. In study II we evaluate the ability of WCS to indicate dose responses and both instruments ability to indicate symptomatic remission during titration with modifiedrelease $\mathrm{MPH}$ and at three months follow-up.

The hypotheses are that (a) PADHD may be used to define remission in adult $\mathrm{ADHD}$, (b) WCS may register direct effects of medication with MPH in adults with ADHD, and (c) WCS may indicate optimal dose levels during titration with modified-release MPH.

\section{STUDY I: METHODS}

\section{Participants}

Study I consisted of 63 participants, 31 men and 32 women, with a mean age of 35.16 years $(S D=11.90)$. Participants had a diagnosis of ADHD at mean age $34.31(S D=$ 11.58) according to the Diagnostic and Statistical Manual of Mental Disorders IV [3]. Behavior ratings of Study I are presented in Table 1. There were no significant differences ( $p s<0.05)$ between men and women with regard to age or any of the behavior rating scales for ADHD. 


\section{Design}

Laboratory assessments of ADHD symptoms before and after clinical treatment with single doses of either shortacting or modified-release formulations of MPH was conducted. Levels of sensitivity and specificity for Prediction of ADHD (PADHD) were investigated using frequency distributions before (no, yes) and after (no, yes) treatment. Fisher's exact test was employed to analyze treatment (baseline, follow-up) and PADHD (no, yes). A mixed design with analyses of variances was used with dependent variables of Hyperactivity, Inattention, Impulsivity and the Weighed Core Symptom scale (WCS). The independent variables were Treatment (before, after) and Gender (men, women).

\section{Instruments}

Qb-Test-Plus. This instrument [19-21] combines an XXtype Continuous Performance Test (CPT) installed as a software program on a PC and an activity test during 20 minutes. While performing the CPT on the computer, movements of the participants are recorded using an infrared Motion Tracking System (MTS) following a reflective marker attached to a head-band. The CPT involves rapid presentation of stimuli involving color (blue, red) and shape (circle, square) on the screen and participants are instructed to press a hand-held button when stimuli repeats itself (a target) and not to press the button when stimuli varies relative to the previous one (a non-target). The stimuli are presented at a pace of one per two seconds, each one visible for 200 milliseconds, and the total number of stimuli is 600 , presented with a $25 \%$ target probability. The purpose of Qb-Test-Plus is to provide objective information regarding cardinal symptoms of ADHD; hyperactivity on basis of motor-activity measured with the camera, and inattention and impulsivity on basis of the CPTtest [19]. Operationalization of variables was done according to a previous study [17]. Hyperactivity was operationalized with the parameter called "distance", i.e., the length (meter) of the path travelled by the headband reflector during the test. Inattention was operationalized as omission errors and impulsivity was operationalized as commission errors.

Weighed Core Symptoms scale (WCS). This scale summarizes the total level of ADHD core symptoms in adults on a scale with ten cut-points ranging from 0 to 100 [17] where 0 indicate maximal amount of and 100 indicate complete absence of ADHD symptoms. The scale is based upon raw scores from the summed and operationalized measures from the Qb-Test-Plus in which the results of hyperactivity have been multiplied with three, inattention with two and impulsivity with one. The ten cut-points of WCS were developed through a procedure previously described [17]. In the present study, WCS correlated (Pearsons' $r$ ) with baseline measures of hyperactivity $(r=0.80, p<0.001)$, inattention $(r=0.62, p<0.001)$, but not with impulsivity ( $p>$ $0.05)$.

Prediction of ADHD (PADHD). This categorical predictor variable [17] regarding ADHD (No/Yes) is based on ADHD behavioral measures from the Qb-Test-Plus (Qscores), i.e., hyperactivity measured in distance, inattention measured with omission errors and impulsivity measured with commission errors. PADHD was developed using qualitative analyses and assessment trials in which the level of sensitivity and specificity was evaluated. The variable generates $86 \%$ sensitivity and $83 \%$ specificity when predicting ADHD versus normative participants from the adult general population, and the probability of PADHD making a correct classification regarding ADHD was 83 and $85 \%$ respectively $[17,18]$.

The Adult Self Report Scale for Adult ADHD v1.1 (ASRS). This screening instrument [22] is derived from the criterions of ADHD in DSM-IV. Part A is the screener and includes the 6 most predictive items while part B holds an additional 12 items, all rated on a five-point scale. The maximum total score for the scale is 72 and 24 for the screener. A value of nine or more on the screener indicates ADHD with high probability [22].

Wender Utah Rating Scale (WURS). This scale [23, 24] retrospectively measures the severance of ADHD symptoms in childhood on a 5 point scale $(0=$ not at all/only a little, $1=$ to some extent, $2=$ a lot, $3=$ severely, $4=$ completely). Adult subjects rate 61 items related to relevant areas, and 25 of the questions are especially sensitive to ADHD [23]. A cut- off of 46 on the 25 -item screener (maximum score of 100) is indicative of ADHD.

Wender Reimherr ADHD Symptoms Scale for Patients (WRASS 1). WRASS [23] estimates symptoms of ADHD from the adult patients point of view on a 5-point scale $(0=$ not at all/only a little, $1=$ to some extent, $2=\mathrm{a}$ lot, $3=$ severely, $4=$ completely). The scale covers areas of attention, hyperactivity, instability of mood, organizational difficulties, sensitivity to stress and impulsivity and is commonly used in Swedish psychiatry with a clinical cut-off of 69 , maximum score is 140 .

Conners Adult ADHD Rating Scale- Short version (CAARSS). This official self-report short-version scale [25] measures 26 items related to four areas of adult ADHD: Inattention, Hyperactivity/restlessness, Impulsivity/emotional lability and problems with self-concept on a four-point scale $(0=$ never, $1=$ sometimes, $2=$ often, $3=$ very often $)$ and the maximum score is 78 .

\section{Procedure}

The current study belongs to a series of investigations performed by the present authors and approved in 2008 by the Regional Ethical Review Board in Uppsala, Sweden (Dnr: 2008/110/2). ADHD-assessments had previously been carried out at the Clinic of neuropsychiatry and CBT, Cereb $(n=51)$, the psychiatric clinic of the County Council in Varmland $(n=11)$ or the NU-health-care psychiatric clinic, all Swedish $(n=1)$. Neuropsychiatric assessments were carried out with similar neuropsychological procedures and included for example retrospective and current medical anamnesis, first- and second part behavior ratings and structured interviews, and psychological tests of memory and attention. In most cases $(n=51)$, assessments included also the unstandardized report of Qb-Test-Plus. Inclusion criterions were 18 to 65 years of age, diagnosis of ADHD according to DSM-IV [3], described chronic course of ADHD symptoms in childhood with some symptoms present before age seven and continue to meet DSM-IV criteria at the time of assessment. 
Table 2. Effects of Prediction of ADHD.

Effects of Prediction of ADHD (No/Yes ADHD), PADHD, for the Baseline and the Treatment condition with a short-acting MPH formulation in a single-dose study including 63 adult participants with ADHD (32 females). The table shows the absolute and relative frequency for PADHD at baseline and during treatment

\begin{tabular}{|c|c|c|c|c|}
\hline & \multicolumn{5}{|c|}{ Prediction of ADHD } \\
\hline & No & & Yes \\
\hline Condition & Frequency & Percent & Frequency & Percent \\
\hline \hline Baseline & 8 & 12.7 & 55 & 87.3 \\
\hline Treatment & 39 & 61.9 & 24 & 38.1 \\
\hline
\end{tabular}

Testing was standardized and performed individually. Participants became seated in front of the PC using a chair without armrests in order to ensure a non-reclining bodyposition. Instructions were given both verbally and with a standardized video [19]. Participants performed a one minutes pre-test to ensure the instructions had been understood correctly. Participants performed the Qb-Test-Plus for 20 minutes in a room with minimal sensory stimuli. Measures were performed at the same day at 103.16 minutes $(S D=$ $55.36)$ from oral intake of MPH $(n=51)$, or some days $(M=$ $13.75, S D=21.55$, range $=1$ to 60 days) after baseline at 193.43 minutes $(S D=122.51)$ from oral intake of MPH $(n=$ 12). Participants were ordinated a short-acting formulation of $10 \mathrm{mg}(n=35)$ or $20 \mathrm{mg}(n=18)$ of MPH, or a modifiedrelease formulation of $18 \mathrm{mg}$ of MPH $(n=9)$ from doctors. One participant was ordinated $54 \mathrm{mg}$ of a modified-release formulation and hence the mean average dosage for the entire sample $(N=63)$ was $13.69 \mathrm{mg}(S D=6.97$, range $=10$ to $54)$. Subjects were informed that enrollment was voluntary and that dropping-out would not interfere with medical treatment or other health-care. The informed consent was signed before any study-related procedures. Participation was associated with travel reimbursement.

\section{STUDY I: RESULTS}

\section{Prediction of ADHD}

Sensitivity and specificity for Prediction of ADHD was investigated using frequency tables for baseline and treatment conditions. Results are presented in Table 2. Fisher's Exact Test (5\% level) indicated most participants in the yesADHD condition before treatment and in the no-ADHD condition after treatment $(p<0.001)$.

\section{Treatment and Gender Differences with Regard to De- pendent Variables}

A mixed Pillai's MANOVA ( 2 x 2 factorial design) was conducted with Treatment (before, after) and Gender (men, women) as independent variables, and Hyperactivity, Inattention and Impulsivity as dependent variables. The analysis revealed significant effects for Treatment $\left(p<0.001, E t a^{2}=\right.$ 0.74 , power $>0.99)$, and Gender $\left(p=0.038, E_{t a}{ }^{2}=0.132\right.$, power $=0.68)$, but not for the interaction Group $\mathrm{x}$ Gender $(p$ $=0.357, E_{t a}{ }^{2}=0.053$, power $=0.28$ ).

Core Symptoms. Univariate F-tests revealed significant effects with regard to Treatment for Hyperactivity $[F(1,61)$
$=115.44, p<0.001]$, Inattention $[F(1,61)=97.19, p<$ $0.001]$, and Impulsivity $[F(1,61)=25.82, p<0.001]$ where the before treatment condition generated lower scores on all variables (indicating higher symptom levels) as compared to the after treatment condition. With regard to Gender, univariate F-tests revealed a significant effect only for Inattention, $[F(1,61)=8.00, p=0.006]$ but not for Hyperactivity or Impulsivity $(p s>0.05)$. Further analyses showed that women had lower scores on Inattention, indicating higher symptom levels, as compared to men. Means and standard deviations are presented in Table $\mathbf{3}$.

WCS. Since the Weighed Core Symptom scale is a composite measure it was analyzed separately with a mixed ANOVA. The analyses showed significant effects for Treatment $[F(1,61)=101.782, p<0.001]$, Gender $[F(1,61)=$ $13.16, p<0.001]$ and for the interaction Treatment $x$ Gender $[F(1,61)=5.80, p<0.05]$. Descriptive analyses showed that the baseline condition was associated with lower scores (indicating higher symptom levels) than the treatment condition, and that men in the treatment condition had higher scores (indicating lower symptom levels) than women in the treatment condition. Regarding the interaction effect, descriptive analyses showed that men gained more improvement from treatment than women. Means and standard deviations are presented in Table $\mathbf{3}$.

\section{STUDY II: METHODS}

\section{Participants}

A total of 30 participants were assessed for eligibility but 20 of them dropped-out, see Table 7. Thus, Study II consisted of 10 participants, 4 men and 6 women, with an ADHD diagnosis according to DSM-IV [3] and whose first MPH- dose was to be initiated and titrated. Behavioral data are presented in Table 6. Participants were outpatients previously diagnosed with ADHD at the psychiatric clinic in the county council of Varmland $(n=8)$ or in the NU-health care psychiatric clinic $(n=2)$. Neuropsychiatric assessments included psychiatric and medical anamnesis, first- and second part behavior ratings and structured interviews, and psychological tests of memory and attention. In four cases, additional tests of cognitive functioning, intelligence, memory and attention were also included. Inclusion criterias were 18 to 65 years of age, diagnosis of ADHD according to DSMIV, described chronic course of ADHD symptoms in childhood with some symptoms present before age seven and continue to meet DSM-IV criteria at the time of assessment. 
Table 3. Descriptive Data for Dependent Variables.

Means $(M)$ and Standard Deviations (SD) for Hyperactivity, Inattention, Impulsivity and Weighed Core Symptoms (WCS) Scale with Regard to Before and after Treatment with Short-Acting or Modified-Release Formulations of MPH with Regard to Men $(n=31)$, Women $(n=32)$ and the Total Group of Adult Participants with ADHD $(N=63)$

\begin{tabular}{|c|c|c|c|c|c|c|}
\hline \multicolumn{7}{|c|}{ Before } \\
\hline & \multicolumn{2}{|c|}{ Men } & \multicolumn{2}{|c|}{ Women } & \multicolumn{2}{|c|}{ Total } \\
\hline & $M$ & $S D$ & $M$ & $S D$ & $M$ & $S D$ \\
\hline Hyperactivity & 27.83 & 23.54 & 24.30 & 20.90 & $26.04 *$ & 22.11 \\
\hline Inattention & 38.14 & 14.21 & 29.19 & 14.99 & $33.60 *$ & 15.18 \\
\hline Impulsivity & 61.20 & 28.55 & 56.92 & 30.87 & $59.02 *$ & 29.59 \\
\hline WCS & $13.23 \propto$ & 16.61 & $6.25 \propto$ & 7.93 & $9.68^{*}$ & 13.32 \\
\hline \multicolumn{7}{|c|}{ After } \\
\hline & \multicolumn{2}{|c|}{ Men } & \multicolumn{2}{|c|}{ Women } & \multicolumn{2}{|c|}{ Total } \\
\hline & $M$ & $S D$ & $M$ & $S D$ & $M$ & $S D$ \\
\hline Hyperactivity & 65.14 & 17.98 & 50.98 & 22.37 & $59.95^{*}$ & 21.39 \\
\hline Inattention & 57.210 & 17.08 & $46.88 \mathrm{a}$ & 15.30 & $51.94 *$ & 16.83 \\
\hline Impulsivity & 77.51 & 23.04 & 70.22 & 23.17 & $73.81^{*}$ & 23.21 \\
\hline WCS & $60.00 \propto$ & 29.44 & $35.00 \propto$ & 30.05 & $47.30 *$ & 32.09 \\
\hline
\end{tabular}

Note. Higher values indicate lower levels of ADHD symptoms; The asterisk $(*)$ indicates significant differences between baseline and follow-up; The square (ळ) indicates significan interaction-effects for gender and condition.

Table 4. Effects of Prediction of ADHD Pre- and Post- Treatment.

Effects of Prediction of ADHD before (No/Yes ADHD) and after (No/Yes ADHD) Treatment

\begin{tabular}{|c|c|c|c|c|}
\hline & \multicolumn{5}{|c|}{ Prediction of ADHD } \\
\hline & No & & Yes & Frequency \\
\hline Condition & Frequency & Percent & 8 & 80 \\
\hline \hline Baseline & 2 & 20 & 1 & 1 \\
\hline Follow-up & 9 & 90 & 20 . & \\
\hline
\end{tabular}

Table 5. Descriptive Data for Dependent Variables at Dose Levels.

Means $(M)$ and Standard Deviations (SD) for Hyperactivity, Inattention, Impulsivity and Weighed Core Symptoms (WCS) Scale with Regard to Dose Levels. Treatment Consisted of a Modified-Release Formulation of MPH in 10 Adults (6 Females) with ADHD

\begin{tabular}{|c|c|c|c|c|c|c|c|c|}
\hline & \multicolumn{2}{|c|}{ WCS } & \multicolumn{2}{c|}{ Hyperactivity } & \multicolumn{2}{c|}{ Inattention } & \multicolumn{2}{c|}{ Impulsivity } \\
\hline & $\boldsymbol{M}$ & $\boldsymbol{S D}$ & $\boldsymbol{M}$ & $\boldsymbol{S D}$ & $\boldsymbol{M}$ & $\boldsymbol{M}$ & $\boldsymbol{S D}$ \\
\hline \hline Baseline & $31.00^{*}$ & 26.85 & $60.30^{*}$ & 25.90 & $34.30^{*}$ & 10.86 & $62.43^{*}$ & 25.38 \\
\hline $18 / 27 \mathrm{mg}$ & 38.750 & 31.82 & 61.57 & 22.22 & 43.04 & 16.35 & 65.71 & 15.54 \\
\hline $36 \mathrm{mg}$ & 55.000 & 28.38 & 68.96 & 11.71 & 52.41 & 23.71 & 74.71 & 14.58 \\
\hline $54 / 72 \mathrm{mg}$ & 77.14 & 24.98 & 73.77 & 15.41 & 61.48 & 11.64 & 87.14 & 5.15 \\
\hline Follow-up & 65.00 & 33.32 & 75.62 & 18.56 & 52.37 & 30.64 & 86.43 & 6.31 \\
& $69.00^{*}$ & 22.34 & $73.88^{*}$ & 11.86 & $55.19^{*}$ & 17.37 & $81.29 *$ & 10.62 \\
\hline
\end{tabular}

Note. Higher values indicate lower levels of ADHD symptoms; The asterisk $(*)$ indicates significant differences between baseline and follow-up; The square ( $(\mathrm{c})$ indicates significant differences from the tabulated value above. 
Table 6. Descriptive Data for Subjective Ratings.

Means $(M)$ and Standard Deviations $(S D)$ of the Behavior Rating Scales of the Present Study, Presented for the Baseline and follow-up Conditions. Treatment consisted of a Modified-Release Formulation of MPH in 10 adults (6 females) with ADHD

\begin{tabular}{|c|c|c|c|c|}
\hline & \multicolumn{4}{|c|}{ Baseline Follow-up } \\
\hline & $M$ & $S D$ & $M$ & $S D$ \\
\hline ASRS-Total & 48.20 & 3.82 & $30.78^{*}$ & 8.87 \\
\hline WRASS & 96.20 & 19.85 & $61.44^{*}$ & 19.18 \\
\hline CAARSS & 55.90 & 13.00 & $36.33 *$ & 11.74 \\
\hline
\end{tabular}

Note. Higher values indicate higher levels of ADHD symptoms; All baseline and the follow-up differences were significant (*) ASRS-T; Adult ADHD Self Rating Scale Total score, WRASS; Wender Reimherr ADHD Symptom Scale, CAARSS; Conners Adult ADHD Rating Scale- Short version.

Table 7. Flow Chart of Study II

\section{Flow Diagram, Study II}

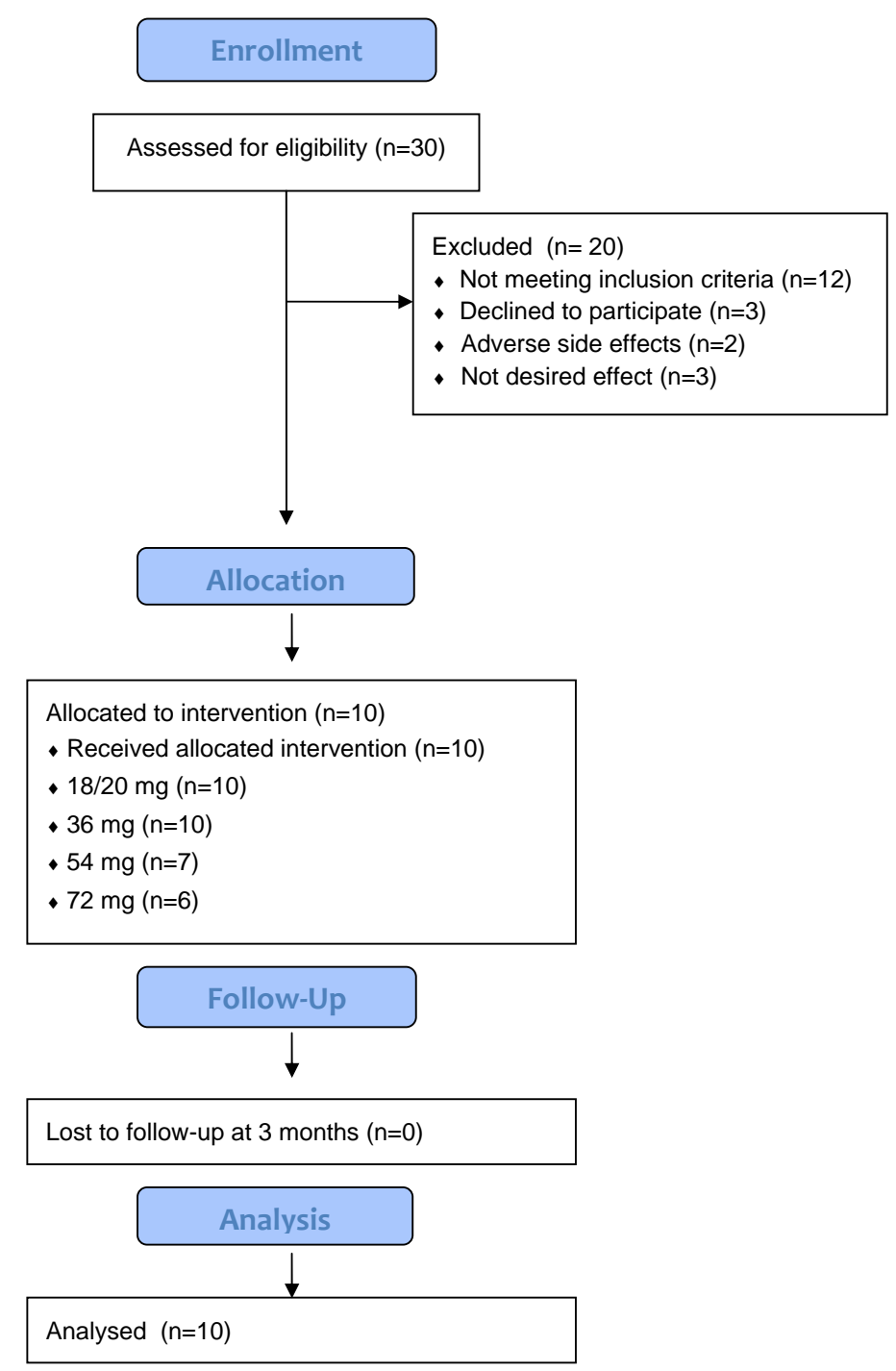

\section{Design}

Pre- and post titration analyses with PADHD (no/yes ADHD) included levels of sensitivity and specificity. De- pendent variables were objective measures of Hyperactivity, Inattention, Impulsivity and WCS. Measures were collected at baseline, for each dose level $(18 / 27,36,54,72)$ and for the 
optimal dose level follow-up at three months. Wilcoxon signed rank test $(5 \%$ level) was used to analyze titration effect upon dependent variables and behavior ratings.

\section{Instruments}

All instruments are presented in the instruments section of Study I.

\section{Procedure}

General study procedures are described in Paper I. Titration time total was individual with one to four weeks interval between each increment of the dose level. Modified-release MPH were ordinated starting at 18 and $27 \mathrm{mg}$ respectively. Study measures were obtained at minimum one to maximum seven days from each increment of the dose level and the psychometric testing generally occurred at 103.16 minutes $(S D=55.36)$ from oral intake. The treatment follow-up was standardized to twelve weeks after the initiation of each patients optimal dose level. Titration was entirely governed by doctors and patients, both blinded from the study measures. Subjects were informed that enrollment was voluntary and that dropping out of the study would not interfere with medical treatment or other health-care. Drop-outs $(N=20)$ are not analyzed in this pilot study, see Table 7.

\section{STUDY II: RESULTS}

\section{Prediction of ADHD}

Sensitivity and specificity for ADHD at pre- and post titration are in Table $\mathbf{4}$.

\section{WCS and Core Symptom Measures at Pre- and Post Ti- tration}

The Wilcoxon signed rank test (5\% level) was employed to assess pre- and post titration conditions with regard to WCS and core symptom measures. WCS was significantly different between the two conditions $(Z=2.82, p=0.005)$. Hyperactivity was not significantly different $(p=0.059)$. However the result could be regarded as a non-significant trend since a Paired-Samples $t$-test (5\% level) yielded a significant effect $[t(9)=-2.34, p=0.044]$. Inattention was significantly different $(Z=2.70, p=0.007)$ and finally, Impulsivity differed between the two conditions $(Z=2.20, p=$ 0.028 ). For means and standard deviations see Table 5.

\section{WCS and Core Symptom Measures During Titration}

The Wilcoxon signed rank test (5\% level) was employed to assess WCS and core symptom measures during titration. Baseline versus the $18 / 27 \mathrm{mg}$ condition yielded significant differences for WCS $(Z=2.25, p=0.024)$ but not for Hyperactivity, Inattention or Impulsivity ( $p s>0.05$ ). The 18/27 versus $36 \mathrm{mg}$ comparison yielded significant differences for WCS $(Z=2.06, p=0.039)$ but not for Hyperactivity, Inattention or Impulsivity ( $p s>0.05$ ). Comparing the 36 vs. 54 $\mathrm{mg}$ and the 54 vs. $72 \mathrm{mg}$ yielded no significant differences for any of the measures $(p s>0.05)$. For means and standard deviations see Table 5.

\section{Subjective Measures of Treatment Response}

A Wilcoxon signed rank test (5\% level) was employed to assess baseline and follow-up conditions with regard to
Wender Adult ADHD Rating Scale (WRASS), the ASRS self-rating scale (ASRSS) and the Conner's Adult ADHD Self Rating Scale Short Version (CARSS). Means and standard deviations are presented in Table 6. WRASS was significantly different between the two conditions $(Z=-2.31, p$ $=0.021)$ and descriptive statistics showed that the baseline condition had a significantly higher mean score than the follow-up condition. The ASRS self-ratings were significantly different $(Z=-2.67, p=0.008)$ and higher at baseline than during follow-up. CAARSS also differed between the two conditions $(Z=-2.67, p=0.008)$ and were higher during baseline than follow-up. It should be noted that higher scores indicates higher levels of ADHD symptoms for all measures whereas higher values of WCS indicates lower levels of ADHD symptoms.

\section{DISCUSSION}

The aim of the present investigation was to evaluate two measures of pharmacotherapy in adult ADHD using shortacting and modified-release formulations of methylphenidate. We hypothesized that (a) PADHD may be used to define remission, (b) WCS may register direct effects of medication with methylphenidate and (c) WCS may indicate optimal dose levels during titration.

The first hypothesis was accepted since in Study I, PADHD correctly classified $87 \%$ of the sample as having ADHD and $62 \%$ of the sample achieved remission. In Study II, $80 \%$ of the sample was correctly classified as having ADHD and $90 \%$ achieved remission. The results of PADHD were validated by the blinding of doctors and patients and were also confirmed by the significant improvements and correlations with behavior ratings, i.e., ASRS, CAARSS and WRASS. It was encouraging that PADHD validated the subjective ratings even though these failed to differentiate dose level improvements.

The second hypothesis was accepted since in Study I, decreased levels of symptoms were registered after low doses of short-acting or modified-release formulations of MPHtreatment. Here, descriptive analyses reported increments of WCS from $9.75(S D=12.27)$ to $47.50(S D=29.75)$. The results of WCS were lower (indicating higher levels of symptoms) than the results of non-ADHD normative participants in previous studies $[17,18]$, i.e., WCS of $63.83(S D=$ $25.72)$ and $67.47(S D=24.07)$, respectively. This indicates that at least some of the participants had not yet gained full effect from their treatment, i.e., being responders rather than remitters, and that their dose level needed adjustment. In Study II, it was evident that the MPH-treatment decreased symptoms $(p=0.005)$ from $31.00(S D=26.85)$ at baseline to $69.00(S D=22.34)$ at follow-up and results are consistent with previous findings of PADHD and WCS [17, 18].

The third hypothesis was accepted since in Study II, symptoms improved from WCS $31.00(S D=26.85)$ to WCS $38.75(S D=31.82)$ at $18 / 27 \mathrm{mg}$ of MPH $(p<0.001)$. Moreover, WCS reported lower symptoms $(M=55.00, S D=$ $28.38, p<.001$ ) when increasing the dose level to $36 \mathrm{mg}$ of MPH. However, WCS did not indicate lower symptoms when increasing the dosage from 36 to $54 \mathrm{mg}(p>.05)$ or from 54 to $72 \mathrm{mg}$ of MPH $(p>.05)$. WCS was investigated 
here in terms of discerning changes in dose levels rather than a dose level response per see. It may be interesting to know that WCS yielded symptom level decrement between baseline and all of the investigated dose levels. But none of the core symptom measures or rating scales was sensitive enough to detect responses to changes in dose level. There were no overall significant differences for gender. Most participants had combined type ADHD with minimal psychiatric comorbidity which may have facilitated the measurement of response and remission as well as contributed to the intervention outcome. The study have several additional limitations, e. g., the low number of participants and especially in Study II, the high level of drop-outs in Study II, the lack of standardized administration of the MPH, the use of both short-release and modified-release formulations of MPH in Study I, the decreased number of participants at the higher dose levels and the use of non-parametric statistics in Study II and the lack of measures for functional improvements, e.g., global assessment of functioning, for comparison with WCS and PADHD are some of the examples of the many limitations of the present study.

\section{CONCLUSIONS}

The present investigation proposed definition and measurement of response and remission using two new instruments for adult ADHD. PADHD and WCS were effective measures of response and remission during pharmacotherapy with short-acting and modified-release formulations of methylphenidate and their ability to calibrate treatment for adults with ADHD was supported. Outcome from prolonged duration of remission and its consequences for the functional ability needs to be given in future studies and the intervention ought to be monitored in terms of global effects on health and psychiatric status. The instruments are proposed as objective tools that may complement behavior ratings and other clinical information in order to monitor therapeutic efficacy in clinical trials and to evaluate intervention studies. WCS and PADHD may also have potential practical implications since it is important for clinicians and patients to have robust and well-defined outcome-criteria in order to evaluate and improve treatment for adult ADHD. Further studies are encouraged in well-being and functional improvements.

\section{AUTHORS' CONTRIBUTIONS}

HE collected and analyzed the material, drafted the manuscript and approved the finalized version. LH designed the study, analyzed the material and approved the finalized version. TN conceptualized and designed the study, analyzed the material, drafted the manuscript and approved the finalized version.

\section{COMPETING INTERESTS}

The authors confirm that this article content has no conflicts of interest.

\section{CONFLICT OF INTEREST}

The authors confirm that this article content has no conflicts of interest.

\section{ACKNOWLEDGEMENTS}

The authors thank all participants. The study was supported by unrestricted grants from the County Council of Värmland, Sweden (98\% of total cost), and Janssen-Cilag AB, Sollentuna, Sweden ( $2 \%$ of total cost). Authors acknowledge the excellent technical assistance of Britt-Marie Hansson, Carolyn Isaksson, Kerstin Ling, Fredrik Ulberstad, Hans Boström, and Petter Knagenhjelm.

\section{REFERENCES}

[1] Frank E, Prien RF, Jarrett RB, et al. Conceptualization and rationale for consensus definitions of terms in major depressive disorder. Remission, recovery, relapse and recurrence. Arch Gen Psychiatry 1999; 48: 851-5.

[2] Kessler RC, Adler L, Barkley R, et al. The prevalence and correlates of adult ADHD in the United Sates: results from the national comorbidity survey replication. Am J Psychiatry 2006, 163: 71623.

[3] Diagnostic and Statistical Manual of Mental Disorders $4^{\text {th }}$ ed. Washington, DC: American Psychiatric Association 2000.

[4] Matza LS, Paramore C, Prasad M. A review of the economic burden of ADHD. Cost Eff Resour Alloc 2005; 3: 5.

[5] Kooij SJ, Bejerot S, Blackwell A, et al. European consensus statement on diagnosis and treatment of adult ADHD: The European Network Adult ADHD. BMC Psychiatry 2010; 10: 67.

[6] Kelsey DK, Summer CR, Casat CD, et al. Once-daily atomoxetine treatment for children with attention-deficit/hyperactivity disorder, including assessment of evening and morning behavior: a doubleblind, placebo-controlled trial. Pediatrics 2004; 114: 1-8.

[7] Spencer T, Biederman J, Wilens T, et al Efficacy of a mixed amphetamine salts compound in adults with attentiondeficit/hyperactivity disorder. Arch Gen Psychiatry 2001; 58: 77582 .

[8] Ramos-Quiroga A, Casas M: Achieving remission as a routine goal of pharmacotherapy in attention-deficit hyperactivity disorder. CNS Drugs 2011; 25: 17-36.

[9] Hasson R, Goldenring Fine J. Gender differences among children with ADHD on continuous performance tests: a meta-analytic review. J Atten Disord 2012 16: 190-8.

[10] Retz W, Rösler M, Ose C, et al Multiscale assessment of treatment efficacy in adults with ADHD: a randomized placebo-controlled, multi-centre study with extended-release methylphenidate. World J Biol Psychiatry 2012; 13: 48-59.

[11] Rösler M, Fischer R, Ammer R, Ose C, Retz W. A randomized, placebo-controlled, 24-week, study of low-dose extended-release methylphenidate in adults with attention-deficit/hyperactivity disorder. Eur Arch Psychol Clin Neurosci 2009; 259: 120-9.

[12] Spencer T, Biederman J, Wilens T, et al. A large, double-blind, randomized clinical trial of methylphenidate in the treatment of adults with attention-deficit/hyperactivity disorder. Biol Psychiatry 2005; 57: 456-63.

[13] Steele M, Jensen PS, Quinn DMP. Remission versus response as the goal of therapy in ADHD: a new standard for the field? Clin Ther 2006; 28: 1892-908.

[14] Swanson J. SNAP-IV scale. Irvine, California: University of California: Irvine Child Development Center 1995.

[15] DuPaul G, Reid R, Power T, Anastopoulos A. ADHD Rating Scale-IV: checklists, norms and clinical interpretations. New York, NY: Guilford Publications 1998.

[16] Guy W. ECDEU: Assessment Manual for Psychopharmacology, Revised. Rockville, MD: US Department of Health, Education and Welfare 1976.

[17] Edebol H, Helldin L, Norlander T. Measuring adult Attention Deficit Hyperactivity Disorder with the Quantified Behavior Test Plus. Psychol J 2013; 2(1): 48-6.

[18] Edebol H, Helldin L, Norlander T. Measuring adult attention deficit hyperactivity disorder with the quantified behaviour test plus. Psychol J 2013; 2(1): 48-62.

[19] QbTech AB. QbTest Plus Clinical Manual. Gothenburg, Sweden, 2010.

[20] QbTech AB. QbTest Plus Technical Manual. Gothenburg, Sweden, 2010 . 
[21] QbTech AB. QbTest Plus Operational Manual. Gothenburg, Sweden, 2010.

[22] Kessler CR, Adler L, Ames M, et al. The world health organization adult ADHD self-report scale (ASRS): a short screening scale for use in the general population. Psychol Med 2005; 35: 245-56.
[23] Ward MF, Wender PH, Reimherr FW. The Wender Utah rating scale: an aid in the retrospective diagnosis of childhood deficit hyperactivity disorder. Am J Psychiatry 1993; 150: 885-90.

[24] Wender PH. Wender AQCC (Adult-Childhood Characteristics) scale. Psychopharmacol Bull 1985; 21: 927-8.

[25] Conners CK, Erhardt D, Sparrow E. Adult ADHD rating scales: Technical manual. Toronto: Multi-Health Systems 1999.

(C) Edebol et al.; Licensee Bentham Open.

This is an open access article licensed under the terms of the Creative Commons Attribution Non-Commercial License (http://creativecommons.org/licenses/by-nc/3.0/) which permits unrestricted, non-commercial use, distribution and reproduction in any medium, provided the work is properly cited. 